\title{
Chinese Educational Image On Screen: A Case Study Of BBC Are Our Kids Tough Enough? Chinese School
}

\author{
Zhemin Chen
}

The Engineering and Technical College of Chengdu University of Technology, Leshan 614000, Sichuan, China

tinaswansea@163.com

\begin{abstract}
The development of Internet technology has enhanced the influence of media. The western mainstream media BBC released the documentary Are our kids tough enough? Chinese school, which has aroused wide attention. As few of the western audience has the experience of Chinese education, the image on the screen would be a significant channel for them to explore how Chinese education works. It provides a platform to reveal the image of Chinese education in other countries, which also indicates the trend and importance of education integration based on the differences between Chinese and western education showed in the documentary.
\end{abstract}

Keywords: Chinese education; Media image; Education integration; Differences between China and western countries

\section{西方镜像中的中式教育一以BBC《我们的孩子足 \\ 够坚强吗？中式学校》为例}

陈哲敏

\author{
成都理工大学工程技术学院, 乐山 中国
}

摘要: 互联网技术的发展进一步增强了媒体的影响力, 西方主流媒体 $\mathrm{BBC}$ 播出的纪录片《我们的孩子足够坚强吗? 中式 学校》引起了广泛的关注。由于西方国家的受众能亲身感知中式教育的人群较少, 片中所呈现的中式教育, 是西方受众了解 与体验中式教育的重要渠道。剖析片中展现的中式教育以及对比中呈现的中西教育差异, 能了解西方国家对中式教育认知的 情况，同时该片本身也是对中西教育融合的尝试，为中西教育国际化融合发展提供新的思路。

关键词: 中式教育; 媒介形象; 教育融合; 中西差异

引言

随着全球化发展, 各国之间联系越来越紧密, 了解他国对中国各方面的认知情况, 是国家发展的诉求, 能有效促进中国国际交流与合作。传播学研究表明如果受众对某议题或事件缺乏丰富的直接经验, 那么他 们对该议题或事件的理解则主要依赖于新闻机构。BBC 纪录片《我们的孩子足够坚强吗? 中式学校 Are our kids tough enough? Chinese school》 (简称为《中式学校》) 以中式教育为话题, 向西方受众展现了 中式教育是如何进行的, 以及中式教育与中国社会之间的联系。

\section{BBC《中式学校》介绍}

英国著名媒体 BBC, 从 2008 年至 2016 年以来拍摄了多部关于中国的纪录片, 从历史文化、美食、自 然风光及社会变迁等多个侧面, 借助镜头形成了对中国国家形象的阐释。 $\mathrm{BBC}$ 纪录片《中式学校》共三集, 每集一小时左右, 正如片中旁白所说 “英国学生在国际竞争中持续落后”、“在国际竞赛名次表上, 英 国学校的排名很不理想, 落后于许多其它欧洲和亚洲国家, 尤其是中国”。片中英国学校的管理层也提到 
“中国学生是我们学生在将来求职时面对的竞争对手。中国正发生着改变, 我们想要确认: 第一, 这个变 化是什么。第二, 是否可以将其融入到我们国家的课堂之中”。中国的教育引起了世界的关注, 西方的教 育在不断变革中探寻与中式教育的融合。五位中国老师在英国以五十名英国学生为教学对象, 要求在整个 教学过程中展现中式教育, 一个月后与英国本土的同年级学生进行测试比赛, 实验的目的是检验哪种教育 方式是最好的。

“传播媒介对外部世界的报道不是 “镜子” 似的反应, 而是一种有目的取舍选择活动。传播媒介根据 自己的价值观和报道方针从现实环境中 “选择” 出他们认为重要的部分或方面进行加工整理, 赋予一定的 结构秩序, 然后以 “报道事实” 的方式提供给受众。” [1] 《中式学校》将为期一个月的教学过程, 通过 “有目的取舍选择”，以纪实的拍摄手法, 浓缩成三个小时左右结构紧凑, 情节跌宕的纪录片。第一集讲 述了实验第一周的教学过程, 第二集展现了师生矛盾不断加深, 第三集聚焦实验最后一周同学们的转变以 及对实验结果的揭晓。由于实验的结果存在悬念, 每集的开头不断营造紧张气氛, 在新旧媒融合的背景下, “互联网和手机作为媒体交互平台推动着 BBC 受众向兼具视听众和网民等多种角色的混合型受众的方向转 化” [2], 受众不仅能通过电视观看《中式学校》, 还可以通过 BBC 网站发表评论, 传播范围扩大, 影响 随之扩大，众多媒体和个人随着情节发展引发了中西方教育的與论热议。

\section{2 《中式学校》镜头中呈现出的中式教育}

第三集最后几分钟揭晓中国老师采用中式教学获得全胜, 但教学中往往伴有文化冲突, 课堂秩序混乱, 学生逃课, 中国老师与英国学生间不和谐的画面。课堂从一度失控到最终学生慢慢理解与逐渐适应中式教 育, 在这转变的过程中, 跟随着镜头, 在片中旁白的带领下, 由一个个课堂内外师生间的互动, 塑造了中 式教育的概貌。

首先, 镜头以信息复现的方式勾勒中式教学的特点, 主要包括长时间学习, 大量的课堂笔记, 激烈竞 争等。强化信息主要借助画面中人物的肢体语言、旁白和教学主体的陈述等。例如在第一集还没有正式上 课之前, 让学生谈谈对中国的了解, 学生提到 “一天要上 12 小时的学, 回家之后还要做五个小时的作业”。 参与实验的 50 名英国学生, 一天的学习也是从 7 点开始, 一位英国学生谈自己的感受时说道 “我不知道 我能不能像中国学生那样, 一直保持这样, 他们起床起得很早, 每天忙个不停”。旁白中也多次提及 “中 国学校的一天是一场 24 小时的马拉松, 7 点就开始了, 午饭时间提前到了 11:30下午还有一次加餐。”、 “还有七个小时才放学”、“第一天才到了下午 $3: 30$, 孩子们就已经有些跟不上节奏了”、“当博航特的 学生放学回家了, 回到家人游戏机和电脑的旁边, 中式学校加餐后学生们还要回到教室。在一天的课程结 束后, 还要上两个小时自习”、“7 点艰苦的一天结束了。”此时, 镜头中有部分学生已经跃在桌子上面 快要睡着了。第三集也有 “学生们在中式学校学习的时间几乎是博航特中学对照组的两倍” 的叙述。十四 岁的 Josh 将电热水壶带进教室泡茶, 以消磨漫长的学时。

再如, “大量的笔记” 在镜头中高频出现。第一集旁白提到 “在中式学校, 学生得到的大多数是要他 们记下来并背下来的笔记” 。英国学生接受采访时说 “我觉得她 (中国老师) 认为我们是机器人, 我们吸 收她给我们的全部知识然后就能全得优, 光从黑板上抄下知识点, 并不能让我真正学会”。另一个学生也 说道 “她上课时学生很容易走神, 在黑板上只是单纯抄她的板书, 这真的很无聊”。虽然画面中对中式教 育学时长, 笔记多的负面评价居多, 但该片也向受众提供了一个开放的思考空间, 对中式教育也有另一种 
看法 “较长的中式教育教学周有一个优势, 即老师们有更多的时间可以与学生建立更紧密的关系”。对于 课堂记笔记, 学生的观点并不一致。有位女学生说道 “我真的很喜欢科学课, 也很喜欢杨老师, 我觉得她 是我最喜欢的老师。我很喜欢写字真的很棒。在不断写的过程当中你就理解了, 那真的很棒我真的很喜欢。” 英国教学主任也说 “学生中有些人很喜欢这个种教学方法, 他们觉得他们不用花太多力气就学会了”。

“严酷的竞争” 也是镜头画面里反复出现的信息。第一集旁白中多次叙述 “这是一个基于高压学习和 残酷竞争的无情学习制度”、“中式教育是基于学校权威, 纪律和残酷竞争的”。第二集中, 旁白介绍 “中 式学校进入了第二周, 五十名十三四岁的学生, 被施以中式教学法, 但截至目前为止, 纪律, 刻苦学习和 竞争的制度不太管用”。体育课上, 两位英国女学生接受采访时说道: “和英国相比中式体育课特别具有 竞争性。”旁白对中式体育课的描述是 “英国体育老师的鼓励方式与中式竞争式的教学形式形成了鲜明的 对比”。第三集在体育课上也有学生谈感受时说: “竞争非常强, 到现在为止我们什么都要和别人比, 几 乎变成了一场迷你比赛。”

其次, 镜头多维度展现中式教育强调学生个人努力的重要性。《中式学校》拍摄的主体不仅仅局限于 五名中国老师与五十名英国学生, 镜头通过英国教师、学校领导及学生家长等的话语交流, 从不同视角呈 现中式教学的一个重要理念即个人努力是提升竞争力与克服困难的利器。英国博航特教学主任与中国老师 赵维之间的对话, 阐释了努力学习能提升个体的竞争力。

中国老师赵维: 将来他们 (英国的学生) 不工作也可以领钱, 他们不需要为生活担忧, 但是在中国一 定要努力学习才可以赚到钱养家。

英国教学主任: 会让大家认为这是我获得良好生活的机会。

中国老师国老师赵维: 比如说现在英国政府正在试图削减福利, 如果他们真的减少的福利。迫使大家 都去工作的话, 他们也许想问题就会从不同的角度了, 如果我获得更好的教育, 竞争力会更强。

英国教学主任: 我不认为我们国家的福利是导致我们的孩子失败的原因, 但也许我们确实不像中国那 样, 有让孩子们努力奋斗成功的绝对动力。

第二集, 实验已过半, 但学生们散漫的态度令中国教师担忧, 家长会上中国老师以演讲的形式与英国 学生父母进行了交流。演讲中老师用客观事实和自己的人生经历, 讲述了在竞争中要不断努力才能适应社 会的发展, 实现自己的目标。镜头里中国老师和英国家长代表着两种不同身份的教学主体, 中国老师陈述 了努力学习的重要性之后, 家长感受到老师们的真诚, 以行动配合老师规范孩子们的行为。

中国老师赵维: 也许有一天你的孩子可能面临和中国的孩子竞争。我不是想说你们的孩子懒, 他们非 常的聪明, 但是这个大环境之下, 他们很多人都过得太安逸了, 有一天当他们遇到外来挑战的时候, 他们 能怎么做。这种挑战我们会遇到, 我们的孩子迟早也会遇到。

中国老师杨君: 我只是从中国小农村走出来的一位中国妇女, 现在能站在你们这些对中国教育有信心, 愿意把你们的孩子送到我们这里的家长面前, 我感到非常的荣幸。目前我还不能确定, 这所中式学校的未 来会如何, 我们是否能取得成功, 纪律的问题使情况变得很糟糕。我们的理念是努力并不代表软弱, 努力 是坚强的象征, 也就是永远不放弃, 一直在努力, 最终达成目标。

第三集镜头里的英国学生也逐渐意识到要通过努力来改变现状。实验的最后一周, 早上七点, 在进教 室之前, 几位学就学习情况进行了交流。通过画面中的对话 “我昨天晚上一整夜都没睡”、“我还不够努 
力”、“我也是, 显然我是负责给博航特 (英国的学校) 丢脸的”, 可以看出英国学生在心理上对中式教 育的看法发生了变化, 之前对中式教育总是排斥与不适应, 现在在潜移默化中从行动上开始认可中式教育, 想要通过努力提高成绩。另一组镜头中, 升旗仪式上, 做演讲的同学对中式学校老师一直提倡的学习要努 力才能有收获的观点表示认可。动员班里的同学都要努力学习 “大家都知道这周后天就要考试了。对此你 们有两个选择选择: 一是不复习, 二是复习。如果你选择前者那就说明你很懒, 你甚至懒得花力气复习。 如果你被难住了, 可以向老师寻求帮助。但是如果你被难住了, 却不去找老师, 因此没通过考试, 就是你 自己的错, 不是老师的错。那么你们会怎么选呢? 复习然后成功通过考试, 还是不复习做个懒鬼, 然后考 试不及格”。成绩出来后, 学生们也证实了自己的努力确实见到了成效。正如学者 Lou jiali 和 Wende1 对中式教育特点进行描述时也提到中式教育中学生要面临许多考试, 学习犹如建造金字塔, 需要坚实的基 础和持之以恒的努力, 中式教育要促进学生们不间断地努力学习。[3]

第三，镜头多场景呈现中式教育关注培养学生的集体意识与集体责任感。在中国习以为常的校园生活 如做早操、升国旗和学生值日等, 在《中式学校》中对英国学生来说是新的体验, 这些场景在镜头中反映 了中式教育相同的主题。

\section{场景一: 做早操}

片中英国学生认为早操是一天当中最美好的时光, 并希望所有的人都能一同体验做早操的美妙感受。 通过采访两位中国老师, 镜头叙述了中式教育中做广播体操不仅能增强学生体质, 更重要的是能培养学生 的团队精神。

中国老师杨君：我喜欢做早操，我小时候就是一个领操员，我喜欢跟着音乐做这些动作。所有的人在 同一个时间做同样的事情，让我们知道我们和其他人是一起的。我们都是团队的一员。

中国老师邹海连: 早操是训练集体意识的一种方式, 我们要一起做事, 而且要正确地一起完成这件事。 场景二: 升国旗

中式教育不仅向学生传授知识, 也关注学生的德育。虽然升国旗场景中英国学生更多地将其认为 “只 是把国旗升到空中而已”，但镜头里中国老师的叙述则表达了升国旗与唱国歌在中式教育中不仅仅局限于 行为本身, 行为的内动力是国家归属感与责任感。

旁白：在中国新的一周以升旗仪式开启。

中国老师：升旗仪式首先是一种表达对祖国的尊重的方式，一种国家归属感。升旗仪式是想提醒你， 你是中国公民, 你有责任为国家服务, 使国家变得更加繁荣富强。每天升国旗, 唱国歌能给人那种责任感。 场景三: 学生做值日

片中中国老师要求英国学生每天要打扫班级卫生, 培养学生的集体意识与集体责任感。镜头中学生们 分工不同, 通过中国老师的叙述传递了中式学校要求学生做值日的目的, 即学生要了解自己的职责, 做好 自己的工作，同时也要学会珍惜集体劳动的成果。

旁白: 中国学校的一切都是为了逐步养成学生的集体责任感。每一天结束时学生们都要打扫自己的教 室。

中国老师赵维: 在中国我们让学生知道自己的职责。打扫教室就是学生的职责所在, 对大多数学生来 说他们会觉得帮助老师是一种荣誉。但是在这里学生们是否意识到了自己的职责呢? 他们对自己的职责有 
所认识吗？当你进入一个社会团体, 他们会告诉你所有你应该承担的责任。你需要付税或者做这样的事情 那样的事情, 如果在此之前你没有让自己的孩子作好准备, 他们怎么能处理好这种转变呢。

\section{3. 结语}

《中式学校》通过制造悬念, 信息复现, 多角度教学主体访谈等方式, 在西方镜像中构建了中式教育 的形象。虽然镜头中着重叙述了英国学生对中式教学的不适应，但镜头中也表达了另一种思想，即教育不 是非此即彼，中西文化不同，社会背景差异较大，因而教育方式也不同，每种教育体制都有其生长的土壤。 镜头中对中式教育的描摹跳出了简单的形式展现, 对于中式教育中长学时, 大量笔记, 激烈竞争等评价发 出了两种声音。镜头也试着深层次挖掘诸如升国旗、做早操、做值日等, 这些被符号化的中式教育所蕴含 的文化内涵。在该片的结尾中西方教师对各自的教学进行了反思, 《中式学校》是一个窗口, 通过中西教 育交流增进了了解, 在中西方教育融合的过程中应相互学习, 取长补短, 不断完善, 最终实现教育与学生 发展、社会发展和世界发展相契合。

\section{4. 致谢}

感谢四川省教育厅, 本文为四川省教育厅科研项目《四川特色文化对外宣传翻译策略研究》(15SB0266) 的阶段性成果之一。

\section{Acknowledgement}

This work was a Project Supported by Scientific Reserch Fund of SiChuan Provincial Education Department (15SB0266).

\section{References}

[1] G. Qingguang, Journalism and communication, Beijing China Renmin University Press , 2011.

[2] L. Shen,Z. Yuning, C. Junxia, et al. china's image on screen, Beijing China Renmin University Press , 2016.

[3] Lou Jiali,Frederick C.Wendel, Preparing for College: Senior High School Education in China,1999, NASSP Bulletin 83(609):57-68. 